\title{
Disseminated cryptococcosis in two boys with novel mutation of CD40 Ligand-Associated X-linked hyper-IgM syndrome
}

\author{
Punchama Pacharn, ${ }^{1}$ Wanatpreeya Phongsamart, ${ }^{2}$ Boonchai Boonyawat, ${ }^{3}$ Orathai Jirapongsananuruk, ${ }^{1}$ \\ Nualanong Visitsunthorn, ${ }^{1}$ Kulkanya Chokephaibulkit ${ }^{2}$
}

\begin{abstract}
X-linked hyper-IgM syndrome (XHIM) caused by CD40L mutations is a primary immunodeficiency condition that increases susceptibility to opportunistic infections. Disseminated cryptococcosis in XHIM is rarely reported in children. Here, we report two related boys who have a novel hemizygous frameshift c.208delC mutation of CD40L. They live in the western region of Thailand and developed disseminated cryptococcosis while receiving regular intravenous immunoglobulin supplementation.
\end{abstract}

Key words: Thailand, disseminated cryptococcosis, novel mutation, CD40 ligand, X-linked hyper-IgM syndrome

\section{From:}

${ }^{1}$ Division of Allergy and Immunology, Department of Pediatrics, Faculty of Medicine Siriraj Hospital, Mahidol University, Bangkok, Thailand

${ }^{2}$ Division of Infectious Diseases, Department of Pediatrics, Faculty of Medicine Siriraj Hospital, Mahidol University, Bangkok, Thailand

3 Division of Genetics, Department of Pediatrics, Phramongkutklao College of Medicine, Mahidol University, Bangkok, Thailand

\section{Corresponding author:}

Punchama Pacharn

Division of Allergy and Immunology, Department of Pediatrics

Faculty of Medicine Siriraj Hospital, Mahidol University

2 Prannok Road, Bangkoknoi, Bangkok 10700, Thailand

E-mail: punchama@gmail.com

\section{List of abbreviations}

ANC absolute neutrophil count

CI confidence interval

CSF cerebrospinal fluid

ECU domain extracellular unique domain

G-CSF granulocyte-colony stimulating factor

GXM glucuronoxylomannan

Hct hematocrit

IVIG intravenous immunoglobulin

MAbs monoclonal antibodies

MIC minimum inhibitory concentration

NETs neutrophil extracellular traps

PHA phytohaemagglutinin

PJP Pneumocystis jiroveci pneumonia

ROS reactive oxygen species

TNFH domain extracellular C-terminal TNF homology domain

WBC white blood cell

XHIM X-linked Hyper-IgM syndrome

\section{Introduction}

X-linked hyper-IgM syndrome (XHIM; OMIM 308230) is a rare primary immunodeficiency disorder, but it is the most common type of hyper-IgM syndrome (HIGM). XHIM is caused by mutations of the CD40 ligand (CD40L; OMIM 300386), which is located at Xq26.3-27.1. ${ }^{1}$ To date, more than 130 mutations have been identified throughout the CD40L. ${ }^{2}$ As a result of the class-switching defect and T-cell dysfunction, patients are susceptible to opportunistic infections (OI), including Pneumocystis jirovecii pneumonia (PJP), Cryptosporidium infection, and infections by members of the herpesvirus family. ${ }^{3}$ Cryptococcus neoformans is a common OI that is mostly found in patients with cell-mediated immuno-deficiency, but it is rarely reported in patients with XHIM.

Here, we report two male cousins with a novel mutation of the CD40L that developed disseminated cryptococcosis while receiving regular intravenous immunoglobulin (IVIG) supplementation.

\section{Report of case}

Patient A was diagnosed with XHIM at 3 years of age. He presented at our center with oroesophageal candidiasis. Immunologic investigations revealed IgG $<7 \mathrm{mg} / \mathrm{dl}$, IgA 17.5 $\mathrm{mg} / \mathrm{dl}, \operatorname{IgM} 431 \mathrm{mg} / \mathrm{dl}, \operatorname{IgE}<3.36 \mathrm{mg} / \mathrm{dl}$, CD4 1,290 cells/ $\mathrm{mm}^{3}$ (35.4\%), CD8 1,158 cells/mm $/ \mathrm{mm}^{3}$ (31.7\%), CD3 2,814 cells/ $\mathrm{mm}^{3}$ (77.13\%), CD19 516 cells/mm $\mathrm{mm}^{3}$ (14.15\%). Lymphocyte proliferation in response to phytohemagglutinin (PHA) was normal.

Patient B, a younger cousin of patient A (Figure 1), was diagnosed with XHIM at 6 months of age. He presented with severe pneumonia and oral ulcer. Immunologic investigations revealed IgG $17.3 \mathrm{mg} / \mathrm{dl}$, IgA $6.86 \mathrm{mg} / \mathrm{dl}, \operatorname{IgM} 37.1 \mathrm{mg} / \mathrm{dl}$, 


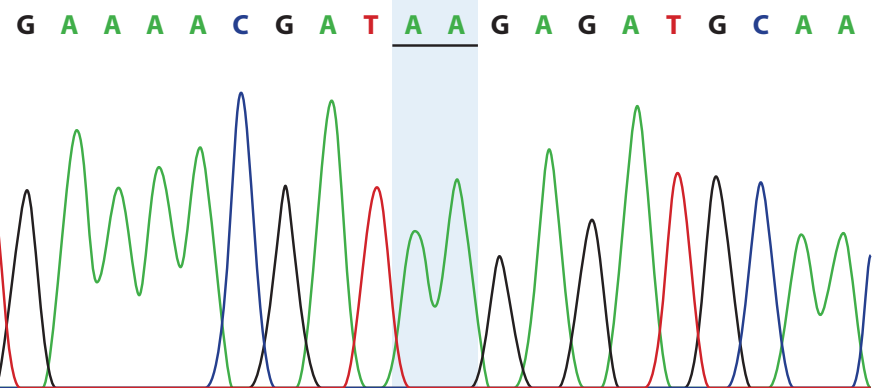

(A) Hemizygous c.208delC mutation in patient DNA

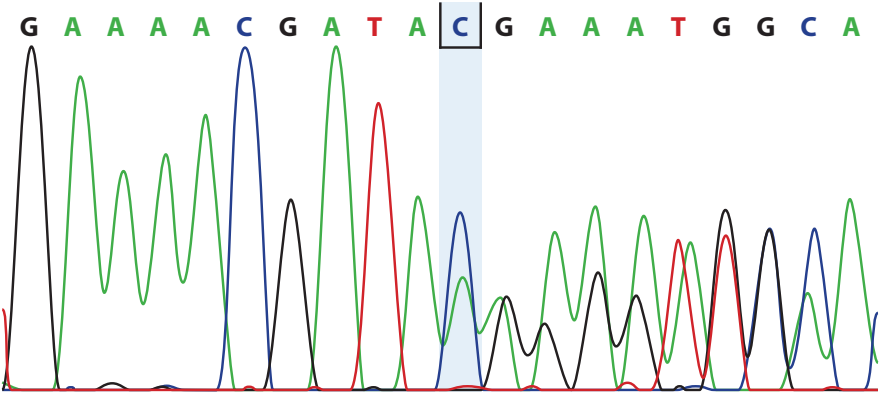

(B) Heterozygous c.208delC mutation in maternal DNA

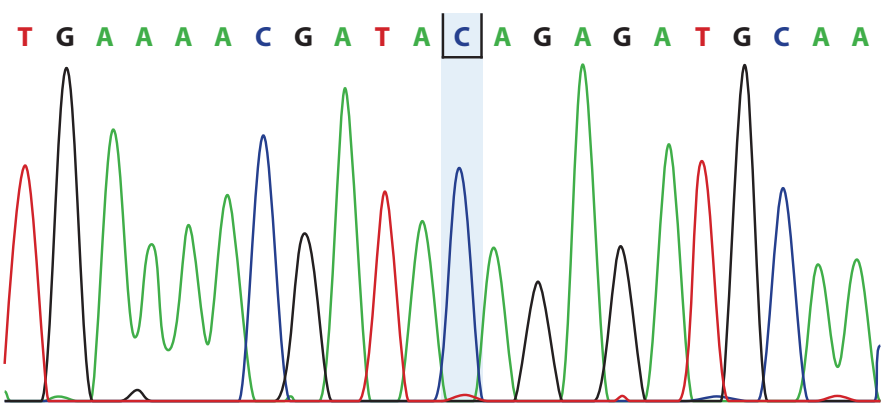

(C) Normal DNA sequence

Figure 1. DNA sequence analysis in exon 2 of the CD40L gene. (A) Direct DNA sequencing revealed a novel hemizygous frameshift c.208delC mutation in patient A whom was earlier diagnosed with X-linked hyper IgM syndrome (XHIGM); (B) A heterozygous c.208delC mutation in the proband"s mother; and, (C) A normal DNA sequence.

$\mathrm{IgE}<4.25 \mathrm{mg} / \mathrm{dL}, \mathrm{CD} 45,889$ cells $/ \mathrm{mm}^{3}(35.9 \%)$, CD8 3,642 cells $/ \mathrm{mm}^{3}$ (22.2\%), CD3 10,401 cells $/ \mathrm{mm}^{3}$ (63.4\%), CD19 4,126 cells $/ \mathrm{mm}^{3}$ (25.15\%). Lymphocyte proliferation in response to PHA was normal.

Since the diagnosis of XHIM, based on the clinical presentations and immunological findings, both patients have been receiving monthly IVIG supplementation and daily trimethoprim-sulfamethoxazole for PJP prophylaxis. IgG trough levels have been consistently above $600 \mathrm{mg} / \mathrm{dL}$ in both patients.

\section{Mutation analysis of the CD40L gene}

After written informed consent was obtained, molecular analysis of the CD40L was performed for the probands and their mothers in 2014. Briefly, genomic DNA was extracted from peripheral blood lymphocytes using commercially available kits according to manufacturer's instructions. Five coding exons and exon/intron junctions of CD40L were separately amplified by polymerase chain reaction (PCR) using previously described primers. ${ }^{4}$ The results revealed a novel hemizygous deletion of cytosine at nucleotide position 208 or c.208delC mutation in exon 2 of CD40L (Figure 1A). This frameshift mutation resulted in a premature termination codon at position 83 (p.Glu70Argfs ${ }^{\star} 13$ ). Molecular analysis of maternal DNA revealed heterozygosity of the same mutation (Figure 1B), which indicates that this mutation was inherited from his mother. The genetic testing of the mother and other available family members was performed. Other affected males and carrier females were identified (Figure 2). The reference sequences were NM_000074 and NP_000065 for CD40L cDNA and amino acid positions, respectively.

\section{Disseminated cryptococcal infection}

At 12 years of age, patient A presented with fever and severe headache. Physical examination revealed signs of meningeal irritation. Laboratory investigations revealed white blood cell (WBC) count 4,050 cells $/ \mathrm{mm}^{3}$ (neutrophils $14.3 \%$, lymphocytes $77.6 \%$ ). The absolute neutrophil count (ANC) was 580 cells $/ \mathrm{mm}^{3}$. Cerebrospinal fluid (CSF) examination revealed WBC 130 cells $/ \mathrm{mm}^{3}$, protein $89 \mathrm{mg} / \mathrm{dl}$, and sugar $<4.32 \mathrm{mg} / \mathrm{dl}$. Encapsulated budding yeasts were identified

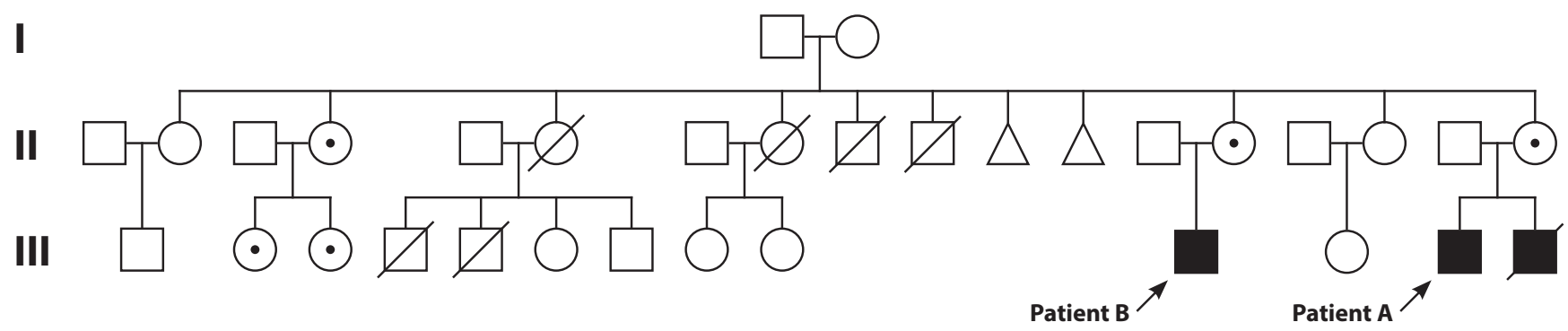

Figure 2. Pedigree of the proband's family. 
by direct examination with India ink. The cryptococcal antigen titer was $>1: 1,024$ in both CSF and serum. Blood and CSF cultures both grew Cryptococcus neoformans. The patient received combination intravenous amphotericin $B(1 \mathrm{mg} / \mathrm{kg} /$ day) and oral fluconazole $(12 \mathrm{mg} / \mathrm{kg} /$ day $)$ treatment. At 4 weeks of treatment, rare encapsulated yeasts were found in the CSF. Amphotericin B was discontinued, but fluconazole was continued for 6 additional weeks for a total of 10 weeks. When that treatment regimen was completed, oral fluconazole was decreased to a maintenance dose of $6 \mathrm{mg} / \mathrm{kg} /$ day for prophylaxis against recurrence. One week after the start of prophylactic treatment, our patient developed recurrent fever and headache. Investigations revealed WBC 2,770 cells $/ \mathrm{mm}^{3}$ (neutrophils $35 \%$, lymphocytes $56 \%$, monocytes $8.8 \%$ ). The ANC was 790 cells $/ \mathrm{mm}^{3}$. The cryptococcal antigen titer was 1:256 in CSF, and $>1: 1,024$ in serum. Intravenous amphotericin B ( $1 \mathrm{mg} / \mathrm{kg} /$ day) and oral fluconazole $(12 \mathrm{mg} / \mathrm{kg} /$ day $)$ were restarted. During hospitalization, the ANC declined to 220 cells $/ \mathrm{mm}^{3}$. He has received G-CSF twice a week since then. After 2 weeks of treatment, the cryptococcal antigen titer in CSF decreased to 1:64. Amphotericin B was discontinued, but oral fluconazole $12 \mathrm{mg} / \mathrm{kg} /$ day was continued at the current dosage. During the subsequent 18-month follow-up, this patient had no recurrent cryptococcal infection while receiving this maintenance dose.

Two months after the onset of illness in patient A, his now 8-year-old cousin (patient B) developed fever with severe headache. Investigations revealed WBC 5,480 cells $/ \mathrm{mm}^{3}$ (neutrophils $11.7 \%$, lymphocytes $66.4 \%$, monocytes $13.5 \%$ ). The ANC was 640 cells $/ \mathrm{mm}^{3}$. CSF examination revealed WBC 280 cells $/ \mathrm{mm}^{3}$, protein $59 \mathrm{mg} / \mathrm{dl}$, and glucose $22 \mathrm{mg} / \mathrm{dl}$. The cryptococcal antigen titer was $>1: 1,024$ in both CSF and serum. His CSF culture grew Cryptococcus neoformans. He received the same antifungal treatment prescribed for patient $A$, followed by maintenance oral fluconazole $12 \mathrm{mg} / \mathrm{kg} /$ day. Patient $B$ had no recurrence of cryptococcal infection during the subsequent 16-month follow-up.

\section{Discussion}

We report 2 boys cousins that harbor a novel mutation of the CD40L, and both have been regularly treated with monthly IVIG and daily trimethoprim-sulfamethoxazole prophylaxis since XHIM diagnosis. In spite of our efforts to avert OI in these two patients, both developed disseminated cryptococcosis within 2 months of each other.

Mutations in the CD40L are highly heterogenous, Missense mutations and small deletions are the main types, accounting for $50-70 \%$ of mutations. ${ }^{5-9}$ We identified a novel hemizygous cytosine deletion at nucleotide position 208 (c.208delC) in exon 2 of the CD40L in our patients. This mutation is predicted to result in premature termination at codon 83 (p.Glu70Argfs ${ }^{\star} 13$ ), which causes truncation of a portion of the extracellular unique (ECU) domain and the entire extracellular C-terminal TNF homology (TNFH) domain, both of which play an important role in the function of CD40L. Based on our review of the literature, this frameshift mutation has never been reported..$^{4-9}$
Cryptococcus neoformans is an encapsulated yeast that is predominantly isolated from pigeon droppings, and from soil contaminated with avian excreta. It causes life-threatening meningoencephalitis mainly in patients with cell-mediated immune deficiency, such as human immune deficiency virus (HIV) infection. ${ }^{10}$ However, it has only rarely been reported in patients with XHIM.

Many factors predispose patients to cryptococcal infection, including host immunologic defect and environmental factors. Although disseminated cryptococcosis can be found in immunocompetent children, ${ }^{11}$ it is more common in patients that have abnormality in cell-mediated immunity. ${ }^{12}$ The patients in this study had normal T-cell counts and normal in vitro T-cell function, as tested by PHA stimulation. However, they may have T-cell dysfunction that is specific to the cryptococcal antigen. In fact, not only $\mathrm{T}$ cell that plays role against host defense to cryptococcal infection, but also humoral and innate immune systems such as macrophages, dendritic cells, neutrophils, natural killer cells, eosinophils. ${ }^{13}$ The role of antibody immunity protection against $C$. neoformans continues to be debated. The most probable action is opsonization for enhanced phagocytosis by macrophages. ${ }^{14}$ Similar to our cases in this report, another study reported disseminated cryptococcal infection in a patient with hypogammaglobulinemia who had normal in vitro T-cell functions. ${ }^{15}$ A previous study showed that patients who produced anticryptococcal antibodies in vivo had relatively better prognosis. ${ }^{16}$ Furthermore, CD40 protein, the receptor of CD40L, is found on B cells, monocytes and dendritic cells. ${ }^{17}$ Therefore, the defect in CD4OL has an effect on the function of these innate immune cells. Taken together, these findings suggest a complex interplay of cellular, humoral, and innate immunity in host defense against cryptococcal infection.

Presence of neutropenia may predispose a patient to develop cryptococcosis. The mechanism of neutropenia is defect in myeloid cell development that is mediated by CD40CD40L interaction. ${ }^{18}$ Neutrophils kill C. neoformans primarily via NADPH oxidase-mediated ROS production, and through the release of neutrophil extracellular traps (NETs). ${ }^{19}$ A previous study in C. neoformans-infected mice found that survival was increased after treatment with G-CSF. ${ }^{20}$

The recommended antifungal treatment for disseminated cryptococcosis is amphotericin B plus flucytosine. However, fluconazole is more widely available and less expensive than flucytosine. In this study, both patients responded well to treatment, but breakthrough infection occurred with lower-dose fluconazole in patient A. Breakthrough infection occurred in $4 \%$ of adults with HIV/AIDS who were receiving fluconazole $200 \mathrm{mg} /$ day (equivalent to $6 \mathrm{mg} / \mathrm{kg}$ / day in children), ${ }^{21}$ and fluconazole at this dosage has been recommended as maintenance suppressive therapy. ${ }^{22} \mathrm{Al}-$ though there is no standard guideline for secondary cryptococcal prophylaxis in primary immunodeficiency patients, we decided to continue the suppressive therapy due to the severity of the disease. It remains unclear whether fluconazole resistance played a role in his relapse of cryptococcal infection despite prescribing the prophylaxis therapy. A recent systematic review of fluconazole resistance found that $24 \%$ of relapsed strain were resistant to fluconazole. ${ }^{23}$ 
Therefore, we decided to increase the maintenance dose to 12 $\mathrm{mg} / \mathrm{kg} /$ day to overcome the possibility of elevated minimum inhibitory concentration (MIC), and there has been no recurrent episode.

In conclusion, patients with XHIM are at increased risk of developing disseminated cryptococcal infection. In settings where flucytosine is not available or accessible, combination therapy consisting of amphotericin B and fluconazole yielded good outcomes. Low-dose fluconazole may not be sufficient to prevent recurrence.

\section{Ethical approval and consent to participate}

Written informed consent was obtained from the parents of the two males profiled herein to report their history and the results of investigations. The reporting of clinical data was approved by the Ethics Committee of the Siriraj Institutional Review Board (SIRB) Faculty of Medicine Siriraj Hospital, Mahidol University, Bangkok,Thailand (COA no. Si 479/2017; approved 4 September 2017).

\section{Consent for publication}

The individual described in this case report completed and signed a consent form authorizing publication and presentation of this manuscript.

\section{Funding}

Siriraj Grant for Research Development, Faculty of Medicine Siriraj Hospital, Mahidol University, Bangkok, Thailand

\section{Authors' contributions}

- PP wrote the manuscript.

- $\mathrm{BB}$ performed genetic testing and wrote the manuscript.

- OJ and NV provided clinical care to the patients.

- WP and $\mathrm{KC}$ revised the manuscript.

- All authors read and approved the final manuscript to be submitted for publication.

\section{Acknowledgements}

The authors gratefully acknowledge Dr. Voravich Leungwedchakarn of the Division of Allergy and Immunology, Department of Pediatrics, Faculty of Medicine Siriraj Hospital for analyzing CD40 ligand expression in the 2 patients profiled in this report.

\section{References}

1. Aruffo A, Farrington M, Hollenbaugh D, Li X, Milatovich A, Nonoyama S, et al. The CD40 ligand, gp 39, is defective in activated $\mathrm{T}$ cells from patients with X-linked hyper-IgM syndrome. Cell. 1993;72:291-300.

2. Notarangelo LD, Peitsch MC, Abrahamsen TG, Bachelot C, Bordigoni P, Cant AJ, et al. CD40lbase: a database of CD40L gene mutations causing X-linked hyper-IgM syndrome. Immunol Today. 1996;17:511-6.

3. Winkelstein JA, Marino MC, Ochs H, Fuleihan R, Scholl PR, Geha R, et al. The X-linked hyper-IgM syndrome: clinical and immunologic features of 79 patients. Medicine (Baltimore). 2003;82:373-84.
4. An Y, Xiao J, Jiang L, Yang X, Yu J, Zhao X. Clinical and molecular characterization of X-linked hyper-IgM syndrome patients in China. Scand J Immunol. 2010;72:50-6.

5. Lee WI, Torgerson TR, Schumacher MJ, Yel L, Zhu Q, Ochs HD. Molecular analysis of a large cohort of patients with the hyper immunoglobulin M (IgM) syndrome. Blood. 2005;105:1881-90.

6. Cabral-Marques O, Klaver S, Schimke LF, Ascendino EH, Khan TA, Pereira PV, et al. First report of the Hyper-IgM syndrome Registry of the Latin American Society for Immunodeficiencies: novel mutations, unique infections, and outcomes. J Clin Immunol. 2014;34:146-56.

7. Wang LL, Zhou W, Zhao W, Tian ZQ, Wang WF, Wang XF, et al. Clinical features and genetic analysis of 20 Chinese patients with X-linked hyper-IgM syndrome. J Immunol Res. 2014;2014:683160.

8. Lee WI, Huang JL, Yeh KW, Yang MJ, Lai MC, Chen LC, et al. Clinical features and genetic analysis of Taiwanese patients with the hyper IgM syndrome phenotype. Pediatr Infect Dis J. 2013;32:1010-6.

9. Gilmour KC, Walshe D, Heath S, Monaghan G, Loughlin S, Lester T, et al. Immunological and genetic analysis of 65 patients with a clinical suspicion of X linked hyper-IgM. Mol Pathol. 2003;56:256-62.

10. Viviani MA, Cogliati M, Esposto MC, Lemmer K, Tintelnot K, Colom Valiente MF, et al. Molecular analysis of 311 Cryptococcus neoformans isolates from a 30-month ECMM survey of cryptococcosis in Europe. FEMS Yeast Res. 2006;6:614-9.

11. Gao LW, Jiao AX, Wu XR, Zhao SY, Ma Y, Liu G, et al. Clinical characteristics of disseminated cryptococcosis in previously healthy children in China. BMC Infect Dis. 2017;17:359.

12. Lizarazo J, Escandon P, Agudelo CI, Castaneda E. Cryptococcosis in Colombian children and literature review. Mem Inst Oswaldo Cruz. 2014; 109:797-804.

13. Hole C, Wormley FL Jr. Innate host defenses against Cryptococcus neoformans. J Microbiol. 2016; 54:202-11.

14. Feldmesser M, Casadevall A. Mechanism of action of antibody to capsular polysaccharide in Cryptococcus neoformans infection. Front Biosci. 1998;3:d136-51.

15. Gupta S, Ellis M, Cesario T, Ruhling M, Vayuvegula B. Disseminated cryptococcal infection in a patient with hypogammaglobulinemia and normal T cell functions. Am J Med. 1987;82:129-31.

16. Diamond RD, Bennett JE. Prognostic factors in cryptococcal meningitis. A study in 111 cases. Ann Intern Med. 1974;80:176-81.

17. Qamar N, Fuleihan RL. The hyper IgM syndromes. Clin Rev Allergy Immunol. 2014;46:120-30.

18. Cabral-Marques O, Takahashi Franca T, Al-Sbiei A, Friederike Schimke L, Khan TA, Feriotti C, et al. CD40 ligand deficiency causes functional defects of peripheral neutrophils that are improved by exogenous IFN-gamma. J Allergy Clin Immunol. [Preprint]. 2018. [cited 2018 Jun 1]. Available from: https://www.sciencedirect.com/science/article/pii/S009167491830318X? via $272 \% 3$ Dihub

19. Chaturvedi V, Wong B, Newman SL. Oxidative killing of Cryptococcus neoformans by human neutrophils. Evidence that fungal mannitol protects by scavenging reactive oxygen intermediates. J Immunol. 1996;156: 3836-40.

20. Graybill JR, Bocanegra R, Lambros C, Luther MF. Granulocyte colony stimulating factor therapy of experimental cryptococcal meningitis. J Med Vet Mycol. 1997;35:243-7.

21. Saag MS, Cloud GA, Graybill JR, Sobel JD, Tuazon CU, Johnson PC, et al. A comparison of itraconazole versus fluconazole as maintenance therapy for AIDS-associated cryptococcal meningitis. National Institute of Allergy and Infectious Diseases Mycoses Study Group. Clin Infect Dis. 1999;28:291-6.

22. AIDSinfo [Internet]. Maryland: U.S. National Library of Medicine [cited 2018 Jun 21].Guidelines for the Prevention and Treatment of Opportunistic Infections in HIV Exposed and HIV-Infected Children; Department of Health \& Human Services; [about 19 screens].Available from: https:// aidsinfo.nih.gov/guidelines/html/5/pediatric-opportunistic-infection/400/ cryptococcosis

23. Bongomin F, Oladele RO, Gago S, Moore CB, Richardson MD. A systematic review of fluconazole resistance in clinical isolates of Cryptococcus species. Mycoses. 2018; 61:290-7 